\title{
Zig-zag version of the Frenkel-Kontorova model
}

\author{
Christiansen, Peter Leth; Savin, A.V.; Zolotaryuk, Alexander
}

Published in:

Physical Review B

Link to article, DOI:

10.1103/PhysRevB.54.12892

Publication date:

1996

Document Version

Publisher's PDF, also known as Version of record

Link back to DTU Orbit

Citation (APA):

Christiansen, P. L., Savin, A. V., \& Zolotaryuk, A. (1996). Zig-zag version of the Frenkel-Kontorova model. Physical Review B, 54(18), 12892-12902. https://doi.org/10.1103/PhysRevB.54.12892

\section{General rights}

Copyright and moral rights for the publications made accessible in the public portal are retained by the authors and/or other copyright owners and it is a condition of accessing publications that users recognise and abide by the legal requirements associated with these rights.

- Users may download and print one copy of any publication from the public portal for the purpose of private study or research.

- You may not further distribute the material or use it for any profit-making activity or commercial gain

- You may freely distribute the URL identifying the publication in the public portal 


\title{
Zig-zag version of the Frenkel-Kontorova model
}

\author{
P. L. Christiansen, A. V. Savin, ${ }^{*}$ and A. V. Zolotaryuk \\ Institute of Mathematical Modelling, Technical University of Denmark, DK-2800 Lyngby, Denmark
}

(Received 2 May 1996)

\begin{abstract}
We study a generalization of the Frenkel-Kontorova model which describes a zig-zag chain of particles coupled by both the first- and second-neighbor harmonic forces and subjected to a planar substrate with a commensurate potential relief. The particles are supposed to have two degrees of freedom: longitudinal and transverse displacements. Two types of two-component kink solutions corresponding to defects with topological charges $Q= \pm 1, \pm 2$ have been treated. The topological defects with positive charge (excess of one or two particles in the chain) are shown to be immobile while the negative defects (vacancies of one or two particles) have been proved at the same parameter values to be mobile objects. In our studies we apply a minimization scheme which has been proved to be an effective numerical method for seeking solitary wave solutions in molecular systems of large complexity. The dynamics of both these types of defects has also been investigated. [S0163-1829(96)03041-X]
\end{abstract}

\section{INTRODUCTION}

The well-known Frenkel-Kontorova (FK) model $^{1-3}$ was originally introduced in the theory of dislocations in solids ${ }^{4}$ to describe the simplest situation when a chain of atoms in crystal is assumed to contain a dislocation, while its crystal environment is modeled by a periodic one-dimensional (1D) substrate potential. Afterwards, this chain model has extensively been used for modeling nonlinear dynamical processes in a variety of condensed matter ${ }^{5-8}$ and biophysical ${ }^{9}$ systems. However, in real physical systems, even in quasi-1D chains (e.g., in biomolecules), besides the longitudinal direction (along a chain), the particles (atoms or molecules) can also move in one or two perpendicular directions and therefore generalizations of the standard (1D) FK model including transverse degrees of freedom are of great interest. In particular, the investigations on the two-dimensional (2D) scalar $^{10-12}$ and vector $^{13,14}$ generalizations of the model, which appear to be very complicated systems, should be mentioned. On the other hand, it is reasonable from the physical point of view to consider also simplified quasi-1D chains where the particles are allowed to move in two directions on a plane. Thus, the Braun-Kivshar (BK) model $^{15}$ has been suggested to describe a chain of atoms interacting via a specific repulsion potential and subjected to the $2 \mathrm{D}$ substrate potential which is periodic in the longitudinal direction and parabolic in the transverse direction. This is the simplest generalization of the 1D FK model, nevertheless, some new aspects of the nonlinear dynamics have been discovered. It was particularly proved ${ }^{15,16}$ that the trivial (linearly arranged) ground state of the chain becomes unstable and it is transformed into a dimerized ground state (the chain takes a zigzag form) when the repulsion between the atoms exceeds a certain critical value. Such a dimerization of the ground state gives rise to the appearance of new topological soliton (kink) states which describe a transition region between two differing chain orientations, but not a vacancy or an excess atom in the chain. The existence of the dimerized ground states and therefore the formation of these "orientational" kinks is due to the linear arrangement of the minima of the substrate potential and of the peculiar form of the intersite interaction, namely pure repulsion. However, many realistic quasi-1D molecular crystals are formed by parallel zig-zag molecular chains. In this case, the zig-zag ground state is formed, in the first turn, by the presence of both the first- and secondneighbor interatomic (or intermolecular) interactions and only then by the interaction of the chain particles with the chain environment. The latter interaction may be modeled by a 2D substrate potential with a zig-zag relief. Then no dimerization effects take place and the topological solitons (kinks and antikinks) that describe only dislocations, can exist in such a system.

The purpose of the present paper is to study a zig-zag generalization of the 1D FK model. The chain particles (atoms or molecules) are supposed to be harmonically coupled via the first- and second-neighbor interactions forming a stable zig-zag structure on a surface even if the chain is considered as an isolated object. ${ }^{17} \mathrm{Next}$, this chain is placed in the planar substrate potential with degenerate minima which exactly coincide with the vertices of a zig-zag backbone (only the commensurate situation is considered in the present paper). Then a certain 1D zig-zag-like "channel", appears in this 2D potential relief, along which the global minima (situated at the zig-zag vertices) and the barriers of a saddle form alternate along the longitudinal direction of the chain. The zig-zag relief with such properties gives rise to the existence of a variety of topological solitons and, at least, two types of them which have differing topological charges, can be treated exactly by using specific numerical techniques.

The paper is organized as follows. In Sec. II, we introduce the zig-zag chain model, describe the $2 \mathrm{D}$ relief of the substrate potential, and derive the corresponding equations of motion. These equations of motion are studied in the next section for small-amplitude oscillations and the linear dispersion law is discussed there. In Sec. IV, we treat analytically two-component kink solutions that describe two types of topological defects. The numerical method for seeking kink profiles is described in Sec. V. In this section, the kink dynamics including different collisions of the defects is also 


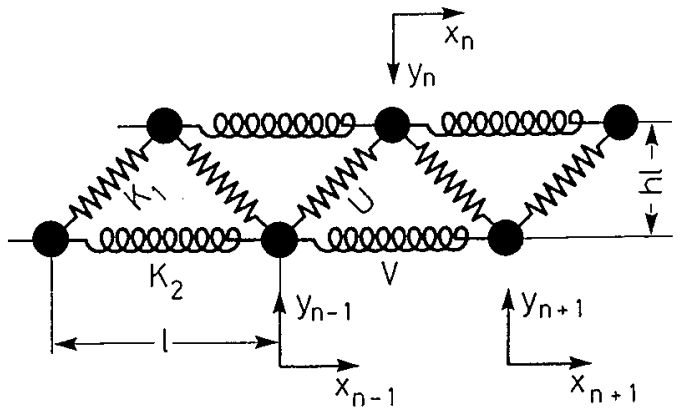

FIG. 1. Schematic representation of the zig-zag chain with $h=1 / 2$.

studied by numerical simulations. Some concluding remarks are outlined in Sec. VI.

\section{A ZIG-ZAG CHAIN MODEL AND A PLANAR ON-SITE POTENTIAL}

Let atoms (or groups of atoms) be linked together in a 2D zig-zag chain, as illustrated in Fig. 1, by the first- and second-neighbor forces with the stiffness constants $K_{1}$ and $K_{2}$, respectively. The chain backbone, with lattice spacing $l$, is directed along the $X$ axis and it can be considered as consisting of two coupled linear chains. Let the molecules of this backbone be situated at the sites $X=n l$, with the integers $n=0, \pm 1, \ldots$ for one of these chains and with the halfintegers $n= \pm 1 / 2, \pm 3 / 2, \ldots$ for the other chain, as shown in Fig. 1. The chain atoms are supposed to have two degrees of freedom (on the $X Y$ plane): the longitudinal $\left(x_{n}\right)$ and transverse $\left(y_{n}\right)$ displacements from the equilibrium positions (vertices of the zig-zag chain). As shown in Fig. 1, the dimensionless parameter $h$ describes the geometry of the chain, namely the thickness of the zig-zag backbone (given in units of lattice spacing $l$ ). Then the equilibrium distance between each pair of the first neighbors is determined by the dimensionless parameter $b=\sqrt{h^{2}+1 / 4}$.

On the other hand, we suppose that the chain is subjected to a $2 \mathrm{D}$ on-site (substrate) potential $Z(u, v)$ with a zig-zag relief, as shown in Figs. 2 and 3. The degenerate global minima of this potential are assumed to coincide exactly with the vertices of the zig-zag chain if it would be isolated from the substrate potential $Z(u, v)$. In other words, when the chain atoms are situated at the vertices of the zig-zag structure, then the interatomic bonds are undistorted and such a configuration forms one of the degenerate ground states of the system (the commensurate situation).

The 2D substrate potential $Z(u, v)$ with a zig-zag relief can be constructed by using a pair of two periodic functions $f(u)$ and $g(u),-\infty<u<\infty$, that satisfy the conditions

$$
f\left(u+\frac{1}{2}\right)=f(u), \quad g(u+1)=g(u) .
$$

Furthermore, both these functions are scaled by their ranges $0 \leqslant f \leqslant 1$ and $0 \leqslant g \leqslant h$ and they are relatively shifted along the $X$ axis with respect to each other in such a way that $f(n / 2)=0, \quad g(n)=0 \quad$ and $\quad f(n \pm 1 / 4)=1, \quad g(n+1 / 2)=h$, $n=0, \pm 1, \ldots$. Particularly, we choose

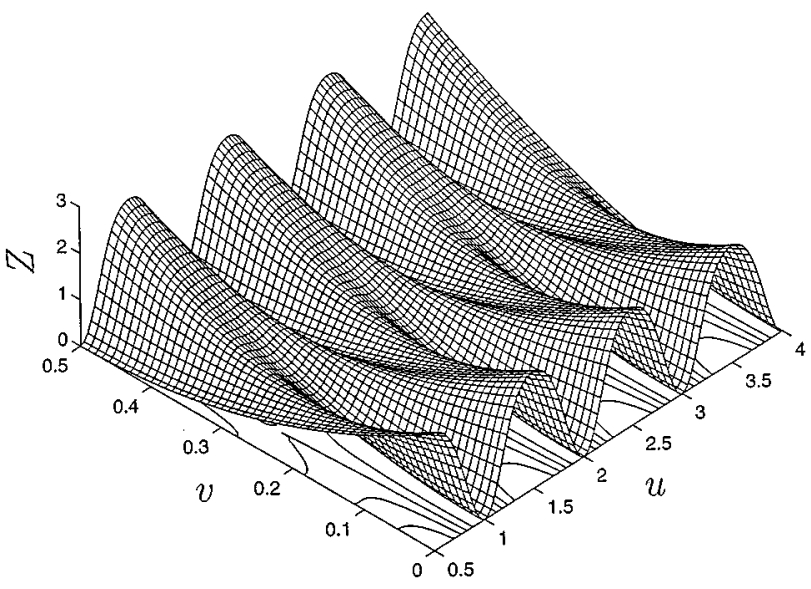

FIG. 2. The 2D substrate potential relief given by the function Eq. (3) with $S=S_{1}$ [see Eqs. (5)], $h=1 / 2$, and $\Omega_{0}=4.47$.

$$
f(u)=\sin ^{2}(2 \pi u), \quad g(u)=h \sin ^{2}(\pi u) .
$$

Then, the zig-zag potential $Z(u, v)$, defined on the $(u, v)$ plane and shown in Fig. 2, can be written as follows:

$$
Z(u, v)=f(u)+\Omega_{0}^{2} S[v-g(u)],
$$

where the function $S(v)$ is assumed to have a singleminimum topology with the minimum at $v=0$ and $\Omega_{0}$ is a characteristic (dimensionless) frequency defined by

$$
\Omega_{0}^{2}=\left.\frac{\partial^{2}}{\partial u^{2}} Z(u, v)\right|_{u=0, v=0}=\left.\frac{\partial^{2}}{\partial v^{2}} Z(u, v)\right|_{u=0, v=0} .
$$

Therefore, according to Eqs. (4), we denote $f^{\prime \prime}(n / 2)=\Omega_{0}^{2}$ where the prime means the differentiation with respect to the argument $u$. It follows from the representation Eq. (3) that $f(u)$ and $g(u)$ may be referred to as "barrier" and "channel" functions, respectively. Several typical examples of the function $S(v)$ can be chosen. In particular, the functions

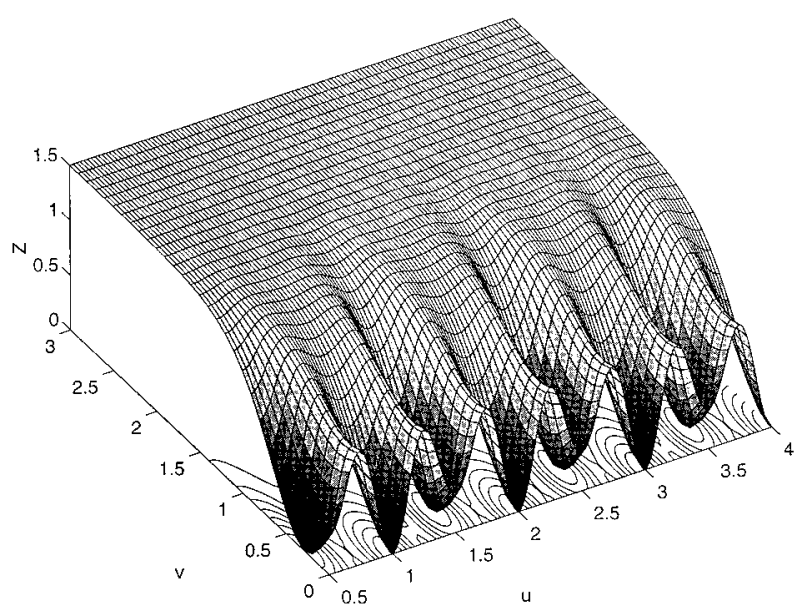

FIG. 3. The $2 \mathrm{D}$ potential relief given by the function Eq. (6) with $T=T_{1}, h=1 / 2 \sqrt{3}$ (corresponding to the zig-zag angle $120^{\circ}$ ), $\Omega_{0}=3.16$, and $\gamma=1.5$. 


$$
\begin{gathered}
S_{1}(v)=\frac{1}{2} v^{2}, \quad S_{2}(v)=\frac{1}{2} \sigma^{2} \sinh ^{2} \frac{v}{\sigma}, \\
S_{3}(v)=\frac{1}{2} v^{2}\left[1-(v / \sigma)^{2}\right]^{-m}
\end{gathered}
$$

with any positive number $\sigma$ and integer $m$, are constructed in analogy with the harmonic, Morse, and Lennard-Jones potentials, respectively. In the limiting case $\sigma \rightarrow 0$, the function $S_{2}(v)$ is reduced to $S_{1}(v)$.

The potential Eq. (3) defined via the functions Eq. (5) is unbounded if $v \rightarrow \pm \infty$. Therefore the representation Eq. (3) can be modified to provide some finite asymptotic values as $v$ tends to infinity, corresponding to the realistic situation. This type of the potentials $Z(u, v)$, shown in Fig. 3, can be represented by

$$
Z(u, v)=f(u)+[\gamma-f(u)] T[v-g(u)]
$$

with some function $T(v)$ satisfying the boundary condition $T( \pm \infty)=1$ and $\gamma=D / \epsilon_{0}>1$ where the constant $D$ may be considered as a dissociation energy. For instance, the functions

$$
T_{1}(v)=\tanh ^{2}\left(\frac{\Omega_{0}}{\sqrt{2 \gamma}} v\right), \quad T_{2}(v)=\frac{v^{2}}{v^{2}+2 \gamma / \Omega_{0}^{2}}
$$

can be chosen as particular examples of the function $T(v)$ normalized according to Eqs. (4).

The total Hamiltonian of the planar zig-zag chain, with the substrate potential $Z(u, v)$ described above, is given by

$H=\sum_{n}\left[\frac{1}{2} M\left(\dot{x}_{n}^{2}+\dot{y}_{n}^{2}\right)+\frac{1}{2} K_{1} l^{2} r_{n}^{2}+\frac{1}{2} K_{2} l^{2} q_{n}^{2}+\epsilon_{0} Z\left(u_{n}, v_{n}\right)\right]$,

where $M$ is the mass of a chain atom (or molecule) and the dot denotes the differentiation with respect to time $t$. According to Fig. 1, the dimensionless deviations from the equilibrium interatomic distances $r_{n}$ and $q_{n}$ are defined by

$$
\begin{aligned}
& r_{n}=\sqrt{\left(\frac{1}{2}+\frac{x_{n+1}-x_{n}}{l}\right)^{2}+\left(h-\frac{y_{n}+y_{n+1}}{l}\right)^{2}}-b, \\
& q_{n}=\sqrt{\left(1+\frac{x_{n+1}-x_{n-1}}{l}\right)^{2}+\left(\frac{y_{n+1}-y_{n-1}}{l}\right)^{2}}-1 .
\end{aligned}
$$

Since $Z(n \pm 1 / 4, h / 2)=1$, the parameter $\epsilon_{0}$ may be referred to as the height of periodic barriers appearing along the channel $v=g(u)$. It is related to the frequency of small-amplitude oscillations of a particle at the minima of the substrate potential $Z(u, v)$ according to $\epsilon_{0}=M l^{2} \omega_{0}^{2} / \Omega_{0}^{2}$.

For the dimensionless description it is convenient to introduce the the dimensionless time and to rescale the spatial variables as follows

$$
\tau={\sqrt{\epsilon_{0} / M}}_{\frac{l}{l}}^{t}, \quad u_{n}(\tau)=\frac{x_{n}(t)}{l}, \quad v_{n}(\tau)=\frac{y_{n}(t)}{l} .
$$

Using Eqs. (9), the corresponding Euler-Lagrange equations of motion take the form

$$
\begin{aligned}
\frac{d^{2} u_{n}}{d \tau^{2}}= & \kappa_{1}\left(u_{n+1}-2 u_{n}+u_{n-1}+\frac{1 / 2+u_{n}-u_{n-1}}{1+r_{n-1} / b}\right. \\
& \left.-\frac{1 / 2+u_{n+1}-u_{n}}{1+r_{n} / b}\right)+\kappa_{2}\left(u_{n+2}-2 u_{n}+u_{n-2}\right. \\
+ & \left.\frac{1+u_{n}-u_{n-2}}{1+q_{n-1}}-\frac{1+u_{n+2}-u_{n}}{1+q_{n+1}}\right)-\frac{\partial}{\partial u_{n}} Z\left(u_{n}, v_{n}\right), \\
\frac{d^{2} v_{n}}{d \tau^{2}}= & \kappa_{1}\left(2 h-v_{n-1}-2 v_{n}-v_{n+1}-\frac{h-v_{n-1}-v_{n}}{1+r_{n-1} / b}\right. \\
& \left.-\frac{h-v_{n}-v_{n+1}}{1+r_{n} / b}\right)+\kappa_{2}\left(v_{n+2}-2 v_{n}+v_{n-2}\right. \\
& \left.+\frac{v_{n}-v_{n-2}}{1+q_{n-1}}-\frac{v_{n+2}-v_{n}}{1+q_{n+1}}\right)-\frac{\partial}{\partial v_{n}} Z\left(u_{n}, v_{n}\right),
\end{aligned}
$$

where $\kappa_{i}=K_{i} l^{2} / \epsilon_{0}, i=1,2$, are the dimensionless stiffness constants of the first- and second-neighbor forces.

\section{SMALL-AMPLITUDE COLLECTIVE EXCITATIONS}

First we consider the linear limit of the equations of motion Eqs. (11) and (12) which describe the behavior of smallamplitude waves in the zig-zag chain. In the harmonic approximation these equations are reduced to
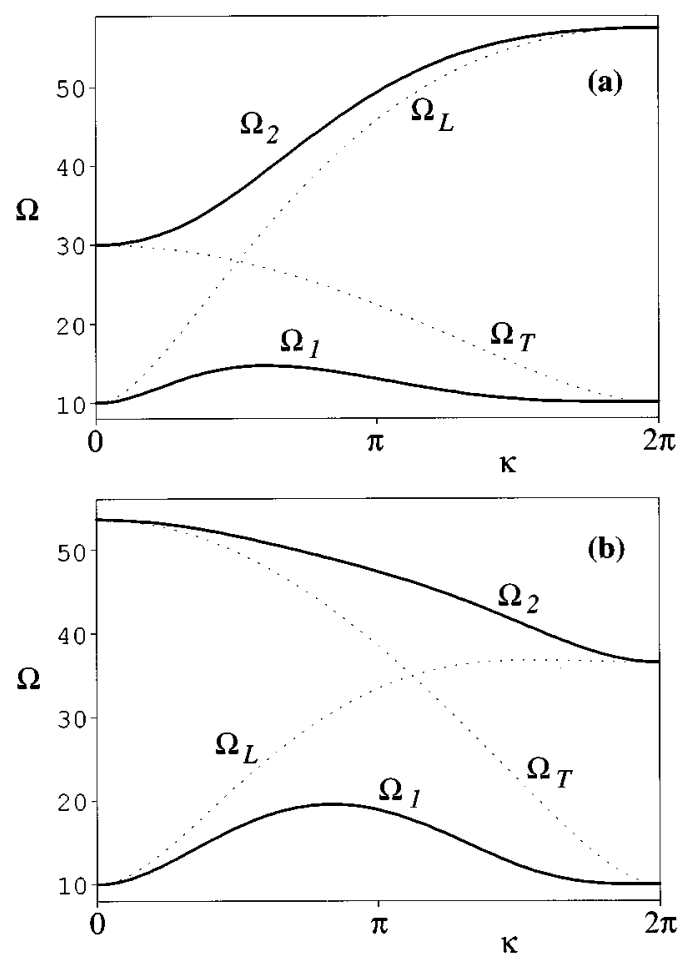

FIG. 4. The frequency spectrum curves $\Omega_{1,2}$ (solid lines) $\Omega_{L, T}$ (dashed lines) for the zig-zag system with the parameters $\Omega_{0}=10$, $\kappa_{1}=1000$, and $\kappa_{2}=100$ : (a) $h=1 / 4$ (the case of positive dispersion of $\Omega_{2}$ at small $k$ ) and (b) $h=3 / 4$ (the case of negative dispersion of $\Omega_{2}$ at small $k$ ). 


$$
\begin{aligned}
\frac{d^{2} u_{n}}{d \tau^{2}}= & \frac{\kappa_{1}}{4 b^{2}}\left(u_{n+1}-2 u_{n}+u_{n-1}\right)+\kappa_{2}\left(u_{n+2}-2 u_{n}+u_{n-2}\right) \\
& -\frac{h}{2 b^{2}} \kappa_{1}\left(v_{n+1}-v_{n-1}\right)-\Omega_{0}^{2} u_{n}, \\
\frac{d^{2} v_{n}}{d \tau^{2}}= & \frac{h}{2 b^{2}} \kappa_{1}\left(u_{n+1}-u_{n-1}\right)-\frac{h^{2}}{b^{2}} \kappa_{1}\left(v_{n-1}+2 v_{n}+v_{n+1}\right) \\
& -\Omega_{0}^{2} v_{n} .
\end{aligned}
$$

Substituting the plane waves

$$
\begin{gathered}
u_{n}=A_{1} \exp [i(k n / 2-\Omega \tau)], \\
v_{n}=A_{2} \exp [i(k n / 2-\Omega \tau)], \quad k \in[-2 \pi, 2 \pi],
\end{gathered}
$$

with arbitrary amplitudes $A_{1}$ and $A_{2}$, the dimensionless wave number $k$, and the frequency $\Omega$, into Eqs. (13), we get the following linear dispersion equation:

$$
\begin{aligned}
& \left(\Omega^{2}-\Omega_{0}^{2}-\frac{\kappa_{1}}{b^{2}} \sin ^{2} \frac{k}{4}-4 \kappa_{2} \sin ^{2} \frac{k}{2}\right) \\
& \times\left(\Omega^{2}-\Omega_{0}^{2}-\frac{4 h^{2}}{b^{2}} \kappa_{1} \cos ^{2} \frac{k}{4}\right)-\left(\frac{h}{b^{2}} \kappa_{1} \sin \frac{k}{2}\right)^{2}=0 .
\end{aligned}
$$

In the decoupling limit, when $\kappa_{1} \rightarrow 0$, the dispersion equation (15) is reduced to the standard linear dispersion law for the chain subjected to a periodic substrate potential:

$$
\Omega^{2}=\Omega_{0}^{2}+4 \kappa_{2} \sin ^{2} \frac{k}{2} .
$$

In the other limit, when $\kappa_{2} \rightarrow 0$ and $h \rightarrow 0(b \rightarrow 1 / 2)$, Eq. (15) is reduced to

$$
\Omega^{2}=\Omega_{0}^{2}+4 \kappa_{1} \sin ^{2} \frac{k}{4}
$$

i.e., to the same dispersion relation [compare with Eq. (16)] if the lattice spacing is rescaled by the substitution $l \rightarrow l / 2$. Note that $\Omega \equiv \Omega_{0}$ is also a solution of Eq. (15) in each of these particular cases.

In the general case, the dispersion equation (15) describes the coupling of the two linear optical-like modes: (i) the longitudinal displacements $u_{n}(\tau)$ with the dispersion law

$$
\Omega_{L}^{2}=\Omega_{0}^{2}+\frac{\kappa_{1}}{b^{2}} \sin ^{2} \frac{k}{4}+4 \kappa_{2} \sin ^{2} \frac{k}{2}
$$

and (ii) the transverse displacements $v_{n}(\tau)$ with the dispersion relation

$$
\Omega_{T}^{2}=\Omega_{0}^{2}+\frac{4 h^{2}}{b^{2}} \kappa_{1} \cos ^{2} \frac{k}{4}
$$

The last term in Eq. (15) appears due to the interaction between these modes. Since it is negative, a gap appears in the frequency spectrum splitting it into two (low- and highfrequency) branches which are given explicitly by the solution of Eq. (15):

$$
\Omega_{1,2}^{2}=\Omega_{0}^{2}+\kappa_{1}\left[1+\left(1-\frac{1}{2 b^{2}}\right) \cos \frac{k}{2}\right]+2 \kappa_{2} \sin ^{2} \frac{k}{2} \mp \sqrt{\left[\kappa_{1}\left(\frac{1}{2 b^{2}}-1-\cos \frac{k}{2}\right)+2 \kappa_{2} \sin ^{2} \frac{k}{2}\right]^{2}+\left(\frac{h}{b^{2}} \kappa_{1} \sin \frac{k}{2}\right)^{2}} .
$$

The upper sign (-) in this solution corresponds to the lowfrequency $\Omega_{1}$ branch, while the other sign $(+)$ gives the high-frequency $\Omega_{2}$ branch of the frequency spectrum. All four dispersion curves $\left(\Omega_{L, T}\right.$ and $\left.\Omega_{1,2}\right)$ in the first Brillouin segment are shown in Figs. 4(a) and 4(b).

In the long-wavelength limit $(k \rightarrow 0)$, the $\Omega_{1}$ branch takes the form

$$
\Omega_{1}^{2} \simeq \Omega_{0}^{2}+\kappa_{2} k^{2}
$$

The other $\Omega_{2}$ branch at $k \rightarrow 0$ is given by the relation

$$
\Omega_{2}^{2} \simeq \Omega_{0}^{2}+\frac{4 h^{2}}{b^{2}} \kappa_{1}+\frac{1}{4}\left(\frac{1}{2 b^{2}}-1\right) \kappa_{1} k^{2}
$$

As follows from the last relation, the long-length waves propagate with positive dispersion if $h<1 / 2$ and with negative dispersion if $h>1 / 2$.

\section{TWO TYPES OF KINK SOLUTIONS}

The system of the coupled equations of motion Eqs. (11) and (12) can be treated both analytically and numerically. To study its soliton solutions analytically, we use the continuum limit which appears in the zig-zag case to be much more crude approximation than in the 1D FK model. Nevertheless, we are still able to get some conclusions about the existence of kink (soliton) solutions. Setting $n=X / l=x$ and substituting the discrete variables $u_{n}(\tau)$ and $v_{n}(\tau)$ by the continuous fields $u(x, \tau)$ and $v(x, \tau)$, respectively, we transform Eqs. (11) and (12) to

$$
u_{\tau \tau}-\left(\frac{\kappa_{1}}{16 b^{2}}+\kappa_{2}\right) u_{x x}+\frac{h}{2 b^{2}} \kappa_{1} v_{x}+\frac{d f}{d u}=\Omega_{0}^{2}[v-g(u)] \frac{d g}{d u},
$$

$$
\begin{aligned}
& 2 \kappa_{1}(h-2 v)\left[1-\frac{b}{\sqrt{\frac{1}{4}\left(1+u_{x}\right)^{2}+(h-2 v)^{2}}}\right] \\
& \quad=\Omega_{0}^{2}[v-g(u)] .
\end{aligned}
$$


(a)

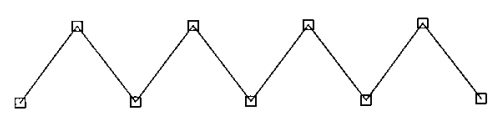

(b)

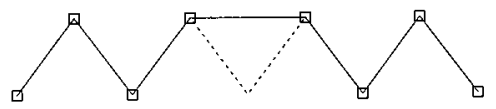

(c)

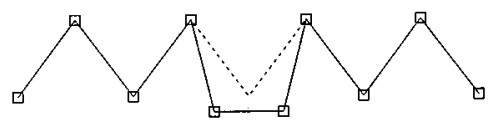

(d)

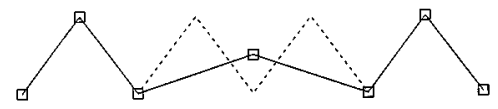

(e)

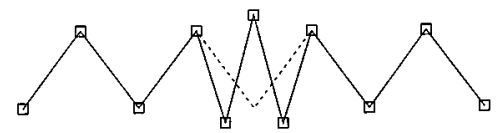

FIG. 5. Schematic representation of the zig-zag chain in (a) the ground state and the topological defects with (b) $Q=-1$ (I-kink), (c) $Q=1$ (I-antikink), (d) $Q=-2$ (II-kink), (e) $Q=2$ (II-antikink).

Here, Eq. (23) has been obtained by linearizing the intersite coupling in Eq. (11), whereas in Eq. (24), both the time and spatial derivatives have been omitted because of the opticallike behavior of the displacement field $v_{n}(\tau)$.

The form of Eqs. (23) and (24) exhibits the existence of two types of two-component kink solutions with differing topological charges. The topological defects described by these kink solutions are schematically shown in Fig. 5 where the ground state [see the regular structure (a) in this figure] of the zig-zag chain is also presented. The kinks (and antikinks) of the first type have the topological charge $Q=\mp 1$ [see Figs. 5(b) and 5(c)] and we call them I-kinks (I-kink if $Q=-1$ and I-antikink for $Q=1$ ). They are defined by the following boundary conditions:

$$
\begin{gathered}
u(-\infty, \tau)=\frac{n}{2}, \quad u(\infty, \tau)=\frac{1}{2}(n \pm 1) ; \\
v(-\infty, \tau)=\frac{1}{2}\left[1 \mp(-1)^{n}\right] h, \quad v(\infty, \tau)=\frac{1}{2}\left[1 \pm(-1)^{n}\right] h,
\end{gathered}
$$

for any integer $n$, where the upper (lower) sign corresponds to a kink (antikink) solution. ${ }^{18}$ The I-kink (with the charge $Q=-1$ ) describes a vacancy in the chain, as illustrated by Fig. 5(b), while the I-antikink (with charge $Q=1$ ) corresponds to an excess particle, as shown by Fig. 5(c). The propagation of the I-kinks is associated with motions of the chain particles approximately in the vicinity of the channel $v=g(u)$. Therefore the right-hand sides of both Eqs. (23) and (24) can be omitted. Then Eq. (24) is easily solved resulting in
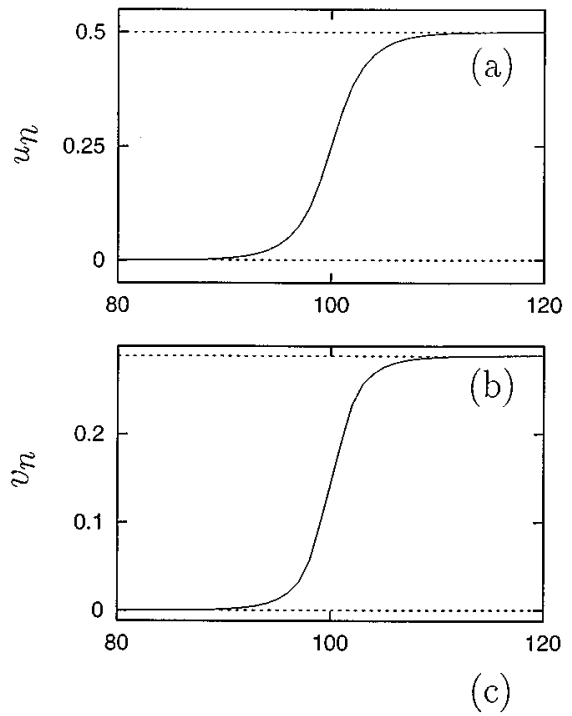

MWMMWWWM

FIG. 6. The standing two-component I-kink $(Q=-1)$ profile $\left(\mathcal{E}=9.1\right.$ and $\mathcal{D}=7.0$ ): (a) longitudinal $u_{n}$ displacements, (b) transverse $v_{n}$ displacements, and (c) the corresponding deformation of the zig-zag chain.

$$
v=\frac{1}{2}\left[h \pm \sqrt{b^{2}-\frac{1}{4}\left(1+u_{x}\right)^{2}}\right],
$$

where the sign $+(-)$ corresponds to the right (left) half of the kink or to the left (right) half of the antikink profile. Expanding the solution (26), we find in the linear approximation that $v \simeq u_{x} / 8 h$ for the left (right) tail and $v \simeq h-u_{x} / 8 h$ for the right (left) tail of the kink (antikink) profile. In this case, Eq. (23) is approximately reduced to

$$
u_{\tau \tau}-c_{1}^{2} u_{x x}+\frac{d f}{d u}=0,
$$

where the characteristic velocity $c_{1}$ is defined by

$$
c_{1}=\sqrt{\kappa_{1} / 16 b^{2}+\kappa_{2}} .
$$

Equation (27) has the standard form that obviously admits kink (antikink) solutions of the typical profile, particularly, the sine-Gordon kinks, propagating with velocities in the segment $0 \leqslant s<c_{1}$. Consequently, both the profiles of the two-component I-kink and I-antikink have the form shown in Figs. 6 and 7 which has been proved below numerically. The above continuum approximation is very crude, but nevertheless, it appears to be sufficient to treat the first type of kinks.

The two-component kinks (II-kinks) of the second type have the topological charge $Q=\mp 2$. They are schematically presented in Figs. 5(d) and 5(e) and satisfy the boundary conditions

$$
\begin{gathered}
u(-\infty, \tau)=\frac{n}{2}, \quad u(\infty, \tau)=\frac{n}{2} \pm 1 ; \\
u(\mp \infty, \tau)=\frac{1}{2}\left[1-(-1)^{n} h\right.
\end{gathered}
$$



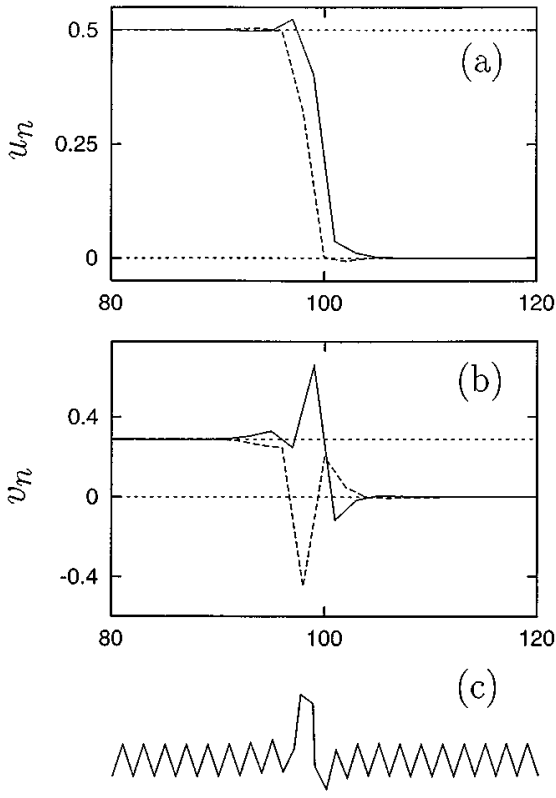

$\eta$

FIG. 7. The same for the standing I-antikink with $Q=1(\mathcal{E}=3.2$ and $\mathcal{D}=2.6$ ). The solid (dashed) lines give the displacements of the chain particles with even (odd) integers $n$.

for any integer $n$, where again the upper (lower) sign corresponds to a kink (antikink) solution. Now the II-kink (with the charge $Q=-2$ ) describes, as shown by Fig. 5(d), a vacancy consisting of two particles in the chain whereas the II-antikink (with the charge $Q=2$ ) corresponds to the defect with two excess particles, as illustrated by Fig. 5(e). In order to treat this type of two-component kinks analytically, we linearize the left-hand side of Eq. (24), assuming the inequality $\left|u_{x}\right| \ll 1$, so that the fields $u(x, \tau)$ and $v(x, \tau)$ can be decoupled as follows:

$$
v=\left(\frac{4 h^{2}}{b^{2}} \kappa_{1}+\Omega_{0}^{2}\right)^{-1}\left[\frac{h}{2 b^{2}} \kappa_{1} u_{x}+\Omega_{0}^{2} g(u)\right] .
$$

Substituting the last relation into Eq. (23), we find the following equation with respect to the field $u(x, \tau)$ :

$$
u_{\tau \tau}-c_{2}^{2} u_{x x}+\frac{d}{d u}\left[f(u)+\frac{1}{2} \frac{g^{2}(u)}{\Omega_{0}^{-2}+b^{2} / 4 h^{2} \kappa_{1}}\right]=0 \text {, }
$$

where the characteristic velocity $c_{2}$ (for the II-kinks) is given by

$$
c_{2}=\sqrt{\frac{\kappa_{1} / 16}{4 h^{2} \kappa_{1} / \Omega_{0}^{2}+b^{2}}+\kappa_{2}} .
$$

Again, Eq. (31) is of the standard form admitting the solutions of the kink type. However, in this case, according to the periodic conditions (1), the effective on-site potential [given by the expression in the square brackets of Eq. (31)] has such a double periodic form that the spatial derivative of the IIkink profile appears to be a two-humped function, as shown
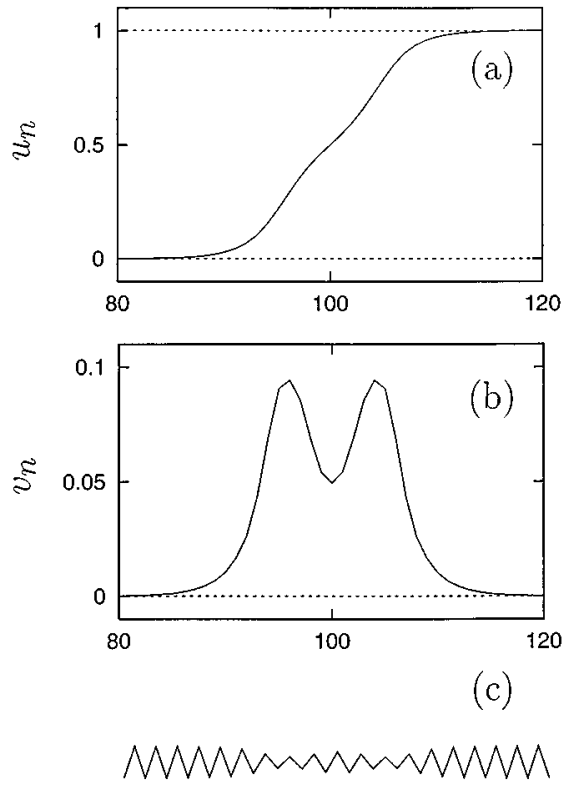

$n$

FIG. 8. The same for the standing II-kink with $Q=-2(\mathcal{E}=19.8$ and $\mathcal{D}=11.3$ ).

in Figs. 8 and 9 where again only numerical solutions are presented. The velocity spectrum of the second-type kinks is $0 \leqslant s<c_{2}$.

\section{A NUMERICAL METHOD}

Both the two types of two-component kink solutions, treated above analytically, can also be found exactly by us-
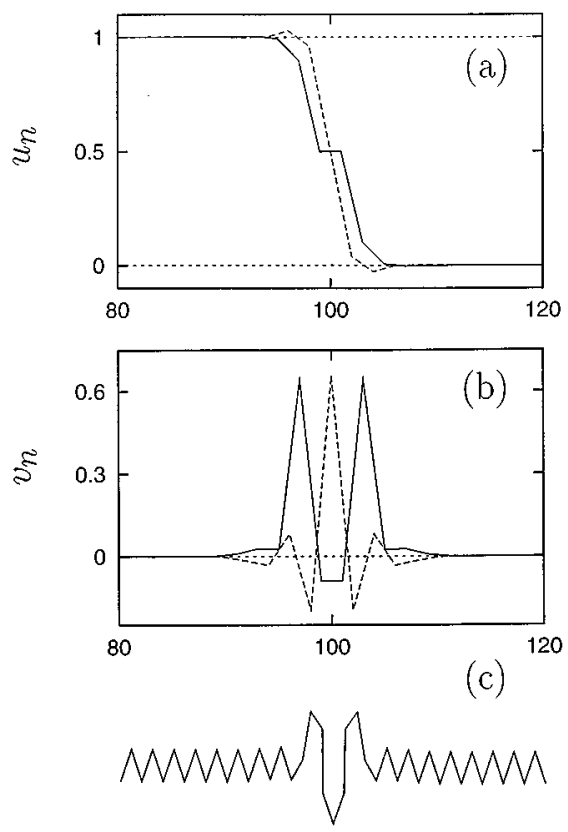

$n$

FIG. 9. The same for the standing II-antikink with $Q=2(\mathcal{E}=6.1$ and $\mathcal{D}=3.8$ ). 
(a)

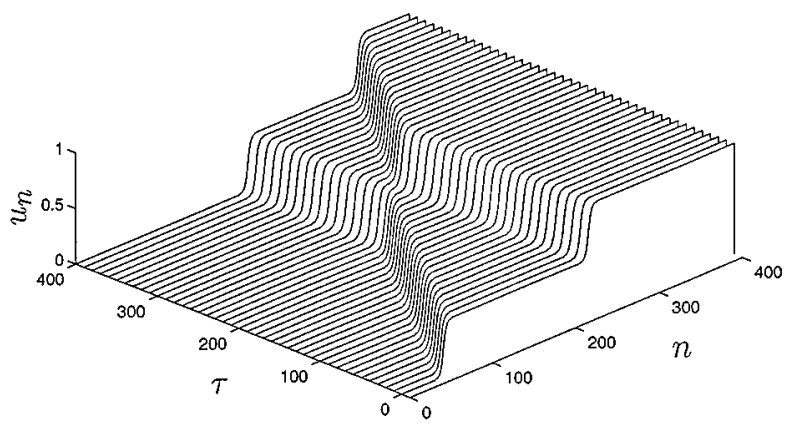

(b)

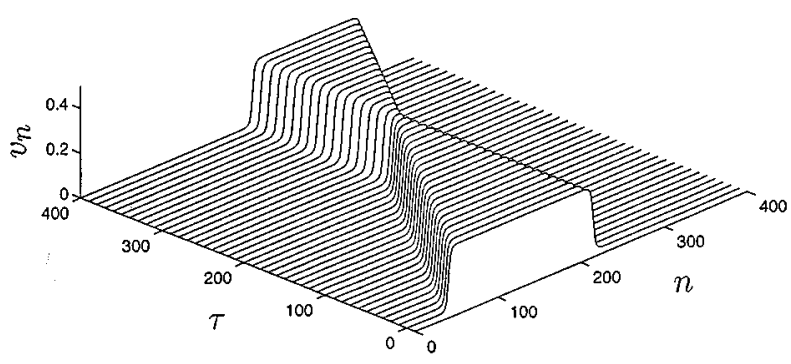

FIG. 10. Elastic collision of the moving (with velocity $s=0.343$ ) kink $(Q=-1)$ with the standing defect of the same charge (I-kink).

ing a numerical minimization scheme which we develop in this section. This scheme seems to be an effective numerical method for seeking soliton solutions in complex molecular systems. Particularly, in the case of the zig-zag chain model, this method allows us to find standing or even moving kink profiles as exact solutions of the basic equations of motion Eqs. (11) and (12). Note that there is another effective method for this purpose which was discovered by Eilbeck and Flesch ${ }^{19}$ and further developed ${ }^{20}$ for variety of nonlinear dynamical systems, including also the 1D FK model. When a (two-component) kink profile has been found by the minimization method, then it can be chosen as an initial condition for numerical simulations of Eqs. (11) and (12). Afterwards, a final profile of the lattice fields $u_{n}(\tau)$ and $v_{n}(\tau)$ obtained under simulations at sufficiently large times $\tau$, allows us to conclude whether or not the initial condition found by the minimization procedure is a correct and stable solution of the equations of motion. The criterion for the method accuracy can be the comparison of a final two-component kink (antikink) profile with the corresponding solution of the minimization procedure when the kink has passed a sufficiently large number of chain sites. Note that we can use for this purpose the cyclic boundary conditions for the lattice fields $u_{n}(\tau)$ and $v_{n}(\tau)$. The main point in such a numerical approach is an appropriate choice of a discrete functional (i.e., a function of many variables) for minimization and, as a rule, such a function can be constructed from the corresponding Lagrangian of the system.

The (dimensionless) Lagrangian that corresponds to the Hamiltonian Eq. (8) has the form (a)

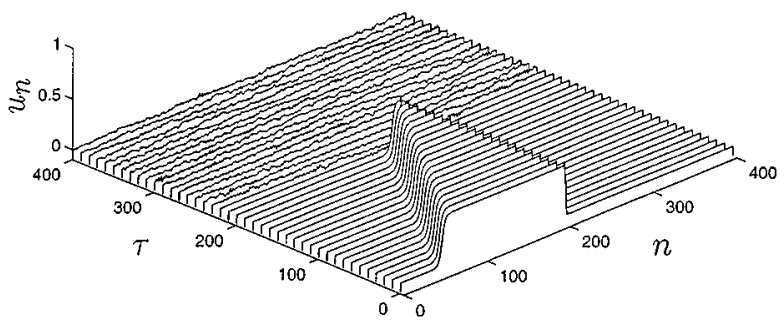

(b)

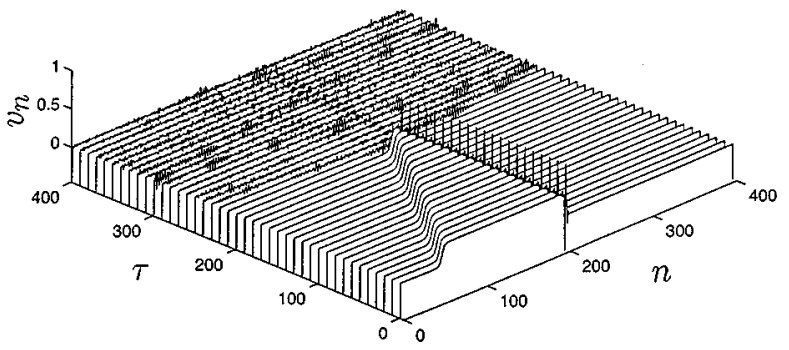

FIG. 11. Recombination of the moving (with velocity $s=0.343$ ) I-kink $(Q=-1)$ under its collision with the standing I-antikink $(Q$ $=1)$.

$$
\begin{aligned}
\mathcal{L}= & \mathcal{L}\left\{\frac{d u_{n}}{d \tau}, u_{n} ; \frac{d v_{n}}{d \tau}, v_{n}\right\}=\sum_{n}\left[\frac{1}{2}\left(\frac{d u_{n}}{d \tau}\right)^{2}+\frac{1}{2}\left(\frac{d v_{n}}{d \tau}\right)^{2}\right. \\
& \left.-\frac{1}{2} r_{n}^{2}-\frac{1}{2} q_{n}^{2}-Z\left(u_{n}, v_{n}\right)\right]
\end{aligned}
$$

where the lattice fields $r_{n}(\tau)$ and $q_{n}(\tau)$ are defined by Eqs. (9) and (10). In order to proceed with obtaining a finitedimensional function for a minimization procedure, we replace the time derivatives $d u_{n} / d \tau$ and $d v_{n} / d \tau$ by appropriate spatial differences of the lattice fields $u(\tau)$ and $v_{n}(\tau)$. Such an approximation can be applied to those lattice functions which (i) are sufficiently smooth from site to site and (ii) have a stationary profile moving with velocity $s$. To do this, we use the simplest approximation as follows:

$$
\begin{aligned}
& \frac{d u_{n}}{d \tau}=-s u^{\prime}(n-s \tau) \simeq-s\left(u_{n+1}-u_{n+1}\right), \\
& \frac{d v_{n}}{d \tau}=-s v^{\prime}(n-s \tau) \simeq-s\left(v_{n+1}-v_{n+1}\right) .
\end{aligned}
$$

Note that in our particular case when a commensurate substrate potential exists, it is not necessary to take into account higher spatial difference derivatives in the expansions (34) which are responsible for the discreteness effects of the chain. However, such an approximation is not valid in the case when the zig-zag chain is considered as an isolated object. ${ }^{17}$ Substituting the expressions (34) into the Lagrangian Eq. (33), we obtain a $2 N$-dimensional function 
(a)

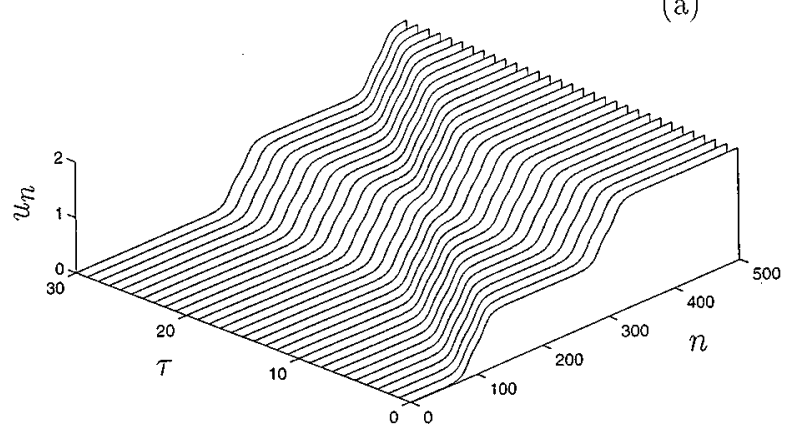

(b)

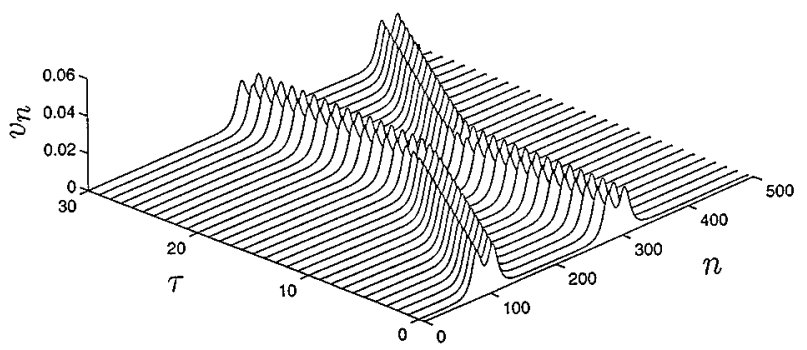

FIG. 12. Collision of the defect of the charge $Q=-2$ (II-kink) with the standing defect of the same charge (II-kink).

$\overline{\mathcal{L}}=\overline{\mathcal{L}}\left(u_{1}, \ldots, u_{N} ; v_{1}, \ldots, v_{N}\right)$ where $N$ is the number of chain particles. Therefore, the minimization problem can be formulated as follows:

$\overline{\mathcal{L}}=\sum_{n}\left[\frac{1}{2} s^{2}\left(u_{n+1}-u_{n-1}\right)^{2}+\frac{1}{2} s^{2}\left(v_{n+1}-v_{n-1}\right)^{2}-\frac{1}{2} \kappa_{1} r_{n}^{2}\right.$

$$
\begin{aligned}
& \left.-\frac{1}{2} \kappa_{2} q_{n}^{2}-Z\left(u_{n}, v_{n}\right)\right] \\
& \rightarrow \min _{u_{2, \ldots, u_{N-1}} ; v_{2}, \ldots, v_{N-1}} \text { or } \max _{u_{2}, \ldots, u_{N-1} ; v_{2}, \ldots, v_{N-1}},
\end{aligned}
$$

where the kink (antikink) boundary conditions at the chain ends

$$
\begin{gathered}
u_{1}=0, \quad u_{N}=1 / 2, \quad v_{1}=0, \quad v_{N}=h \quad \text { (I-kink) } Q=-1, \\
u_{1}=1 / 2, \quad u_{N}=0, \quad v_{1}=h, \quad v_{N}=0 \quad \text { (I-antikink) } Q=1, \\
u_{1}=0, \quad u_{N}=1, \quad v_{1}=v_{N}=h \quad(\text { II-kink) } Q=-2, \\
u_{1}=1, \quad u_{N}=0, \quad v_{1}=v_{N}=0 \quad \text { (II-antikink) } Q=2,
\end{gathered}
$$

are supposed to be fixed under the minimization process. Note that in the continuum limit, the latter conditions correspond to Eqs. (25) and (29). A solution to the problem Eq. (35) is identified with a kink (antikink) profile and it can be found, for instance, by using the steepest descent method.

In order to be certain that the kink solution corresponds to a minimum (or maximum) of the Lagrangian Eq. (33), we substitute the second-order time derivatives $d^{2} u_{n} / d \tau^{2}$ and (a)

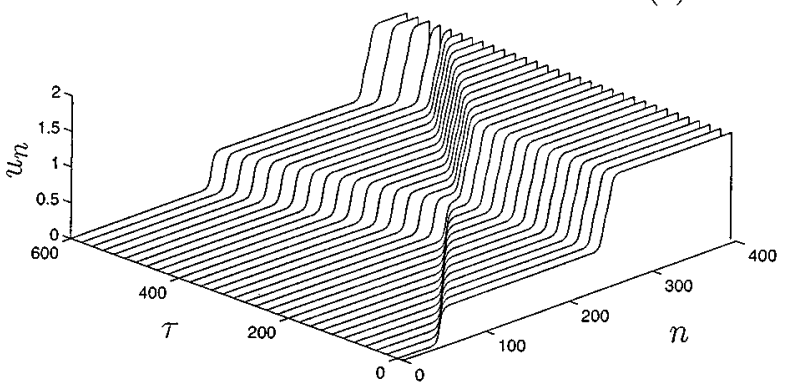

(b)

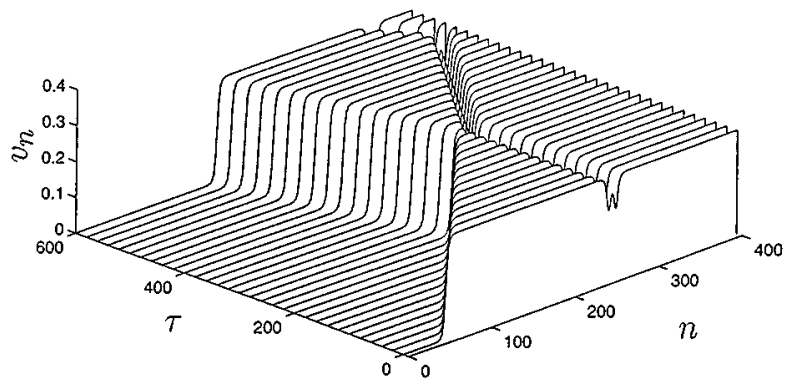

FIG. 13. Elastic collision of the moving (with velocity $s=0.343$ ) defect of the charge $Q=-1$ (I-kink) with the standing defect of the charge $Q=-2$ (II-kink).

$d^{2} v_{n} / d \tau^{2}$ by the second-order spatial difference derivatives $s^{2}\left(u_{n+2}-2 u_{n}+u_{n-2}\right)$ and $s^{2}\left(v_{n+2}-2 v_{n}+v_{n-2}\right)$, respectively. In other words, we accomplish the similar approximation procedure as was done by using Eqs. (34). The resulting difference equations are nothing more than the extrenum conditions $\partial \mathcal{L} / \partial u_{n}=0$ and $\partial \overline{\mathcal{L}} / \partial v_{n}=0$. Of course, solving the minimization problem Eq. (35), we may loose some soliton solutions which correspond to saddle points of the Lagrangian $\overline{\mathcal{L}}$. Therefore we have examined this possibility by "deforming" the Lagrangian surface $\overline{\mathcal{L}}$ in such a way that the saddle points are transformed into minima, similarly to the procedure performed for an isolated zig-zag chain, ${ }^{17}$ and then by minimization of the deformed function. This procedure did not exhibit new soliton profiles differing from those found by solving the problem Eq. (35).

The results of the solution of the minimization problem Eq. (35) are presented in Figs. 6-9 where the kink (and antikink) profiles of both types are plotted. The accuracy of the (moving) kink profiles, found by the minimization procedure and taken as initial conditions for solving the equations of motion Eqs. (11) and (12), was examined from their comparison with the final profiles obtained at those times when the kinks have passed 100000 chain sites. A perfect coincidence of the initial and final profiles was observed. As follows from the comparison between Figs. 6 and $7(Q=\mp 1)$ and between Figs. 8 and $9(Q=\mp 2)$, at the same parameter values, both the I- and II-kinks (vacancies of one or two atoms) have broad profiles while the corresponding antikinks (excess one or two atoms in the chain) are quite narrow objects. Therefore the positive topological defects $(Q=1,2)$ appear to be immobile (pinned) objects while the negative defects can propagate along the zig-zag backbone retaining 
(a)

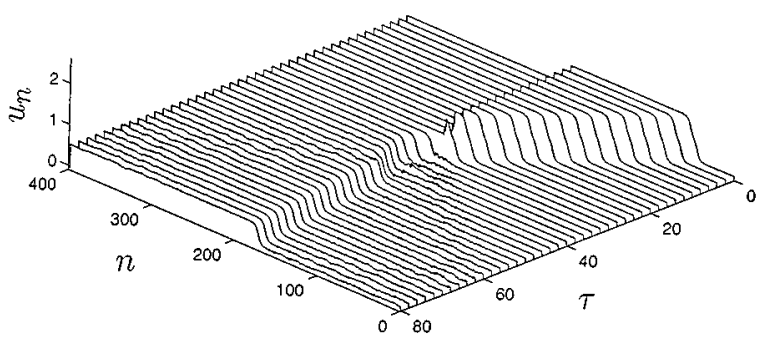

(b)

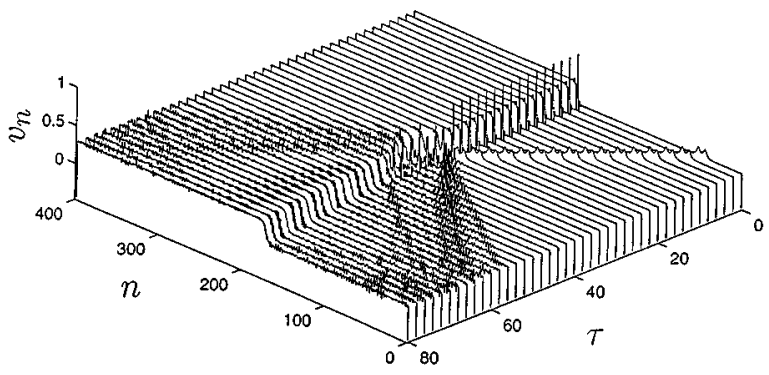

FIG. 14. Creation of the defect of the charge $Q=-1$ (I-kink) under the collision of the moving (with velocity $s=0.352$ ) defect of the charge $Q=-2$ (II-kink) with the standing defect of the charge $Q=1$ (I-antikink).

their velocity and profile. Such breaking the kink-antikink symmetry is absent in the 1D FK model. In a zig-zag chain, it appears due to the effective ("geometric") anharmonicity of the intersite coupling caused by the zig-zag structure.

In order to describe and compare more precisely the properties of the kink solutions obtained numerically, it is convenient to calculate the dimensionless kink energy

$$
\begin{aligned}
\mathcal{E}= & \mathcal{E}(s)=\sum_{n=1}^{N}\left[\frac{1}{2} s^{2}\left(u_{n+1}-u_{n-1}\right)^{2}+\frac{1}{2} s^{2}\left(v_{n+1}-v_{n-1}\right)^{2}\right. \\
& \left.+\frac{1}{2} \kappa_{1} r_{n}^{2}+\frac{1}{2} \kappa_{2} q_{n}^{2}+Z\left(u_{n}, v_{n}\right)\right]
\end{aligned}
$$

the position of the kink center

$$
N_{c}=\frac{1}{2}+\sum_{n} \frac{u_{n+1}-u_{n}}{u_{N}-u_{1}}
$$

and the kink width

$$
\mathcal{D}=\mathcal{D}(s)=2 \sqrt{\sum_{n}\left(n-N_{c}\right)^{2}\left(u_{n+1}-u_{n}\right) /\left(u_{N}-u_{1}\right)} .
$$

Then, for instance, the standing kink with the charge $Q=-1$ has the width of seven chain sites, i.e., $\mathcal{D}(0)=7.0$. This kink can propagate with velocities in the segment $0 \leqslant s \leqslant 0.343$. At higher velocities, its motion is accompanied by emission of small-amplitude waves and gradual decreasing velocity to this value. Afterwards, the kink propagation becomes stable. (a)

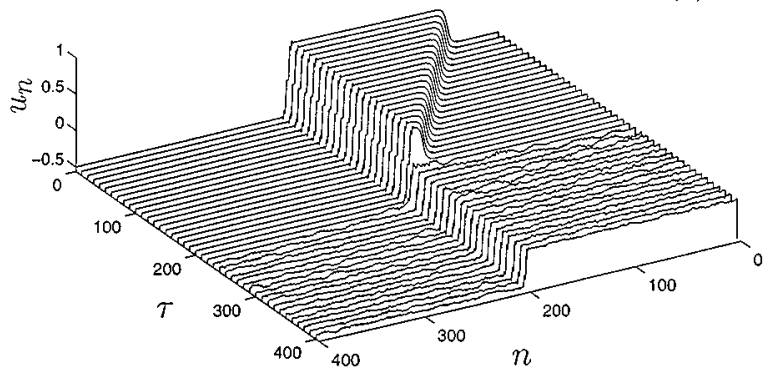

(b)

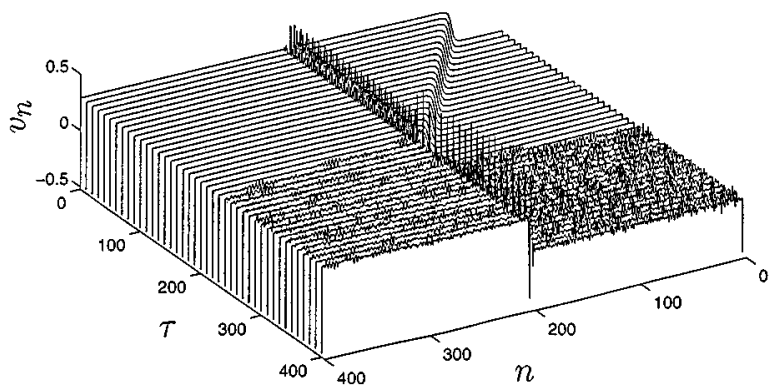

FIG. 15. Creation of the defect of the charge $Q=1$ (I-antikink) under the collision of the moving (with velocity $s=0.343$ ) defect of the charge $Q=-1$ (I-kink) with the standing defect of the charge $Q=2$ (II-antikink).

The same is true for the kinks with the charge $Q=-2$. This kink has the width $\mathcal{D}(0)=11.3$ and its velocity spectrum is $0 \leqslant s \leqslant 2.352$.

It should be noticed that the energy of a II-kink exceeds the energy of two I-kinks. Therefore a pair of I-kinks should be a more favorable state than the II-kink state. However, simulations of their evolution have shown that the II-kink is dynamically stable and it does not decay into two separate I-kinks.

Both the positive defects (antikinks with $Q=1,2$ ) are narrow objects, forming the pinned states. Thus, for $Q=1$ we have $\mathcal{E}(0)=3.2$ and $\mathcal{D}(0)=2.6$, while for the second type $(Q$ $=2$ ) the corresponding values are $\mathcal{E}(0)=6.1$ and $\mathcal{D}(0)=2.8$. Note that in this case, $\mathcal{E}_{+2}<2 \mathcal{E}_{+1}$ and therefore the defect with $Q=2$ is more energetically favorable than two separate defects with $Q=1$. As for the dependence of the kink energy and width on the velocity $s$, we have proved the similar behavior as in the 1D FK model, i.e., the kink energy $\mathcal{E}(s)$ is a monotonically increasing function, while the kink width $\mathcal{D}(s)$ decreases with increasing the velocity $s$.

Using the results of the minimization procedure as initial conditions for simulations of the equations of motion Eqs. (11) and (12), we have studied the whole variety of the collision processes between both the types of topological defects. These results are presented in Figs. 10-15. Thus, Fig. 10 demonstrates the elastic interaction of the I-kinks (vacancies) of the same topological charge $Q=-1$, while Fig. 11 shows the recombination of the vacancy $(Q=-1)$ and the defect with an excess particle $(Q=1)$. Figure 12 illustrates the interaction between the II-kinks of the same charge $Q=2$ 
which appear to be sufficiently extended objects. The elastic collision process of the negative I- and II-defects is shown in Fig. 13. The dynamical processes of "recharging" the defects with differing topological charges have also been studied. Thus, the processes of the creation of the defects with the charges $Q=\mp 1$ (I-kink and I-antikink) are illustrated by Figs. 14 and 15.

\section{DISCUSSION}

We have suggested and studied both analytically and numerically a zig-zag version of the standard (1D) FK chain model which may be considered as one of its reasonable generalizations including both (i) transverse degrees of freedom and (ii) second-neighbor interatomic interactions. This model may also be considered as a modified version of the Braun-Kivshar model. ${ }^{15}$ Compared to the BK model, which seems to be more specific with possible applications to the dynamics of adsorbed atoms on crystal surfaces (due to the presence of a repulsive interparticle interaction),${ }^{8}$ our generalization is more straightforward. Indeed, similarly to the 1D FK model, our zig-zag chain can also be found in the stable ground state if it is isolated from the planar substrate because both attractive and repulsive (harmonic) forces are involved into the interatomic (intermolecular) interactions. However, such an equilibrium configuration can exist only in the case when the secondary structure of a chain is taken into account. In the simplest case, the secondary structure can be realized by means of second-neighbor interactions and, as a result, a stable ground state of an isolated zig-zag chain is formed. ${ }^{17}$ Note, in order to have a stable ground state for an isolated 1D chain, it is sufficient to consider only nearestneighbor couplings. Next, the zig-zag chain backbone (with both the primary and secondary structures), as a stable object (e.g., a macromolecule), was subjected to a $2 \mathrm{D}$ on-site periodic potential of the zig-zag-like relief. In the present paper, we have restricted ourselves to the commensurate situation when the vertices of the zig-zag chain exactly coincide with the global degenerate minima of the on-site potential.

As should be expected, the existence of transverse degrees of freedom drastically changes the dynamics of 1D lattices (both isolated and subjected to a substrate). As for the case with the presence of a periodic substrate, the whole variety of soliton solutions depends on the type of a substrate potential. Thus, while in the case of the BK model, two types of kinks ("massive" and "nonmassive") exist, in our model only "massive" kinks are possible, but they themselves appear to be of two types describing a vacancy which consists of one or two particles (I- or II-kink), or an excess of one or two particles (I- and II-antikink). ${ }^{18}$ The further comparison of the BK model with our zig-zag model shows such a common property as breaking symmetry between the kink and antikink solutions. Similarly to the studies, ${ }^{15,16}$ in our case, vacancies of both types (I- and II-kinks) appear to be mobile defects, while excess particles (I-, II-antikinks) are immobile and become pinned defects. This kind of kink-antikink asymmetry is due to the presence of the geometric anharmonicity in the intersite coupling that exists even if both the first and second neighbor are of the harmonic type. The experimental situations when the mobility of defects crucially depends on their polarity are known, for instance, in ice physics, ${ }^{21}$ and therefore the zig-zag modeling, particularly, for the proton transport in hydrogen-bonded crystals and biomolecules, ${ }^{22,23}$ is of great interest.

\section{ACKNOWLEDGMENTS}

This work was partially carried out with the financial support from the European Economic Community (EEC) under the INTAS Grant No. 94-3754. A.V.Z. would also like to express his gratitude to The MIDIT Center and The Institute of Mathematical Modelling of The Technical University of Denmark for the Guest Professorship and hospitality. Stimulating and useful discussions with T. C. Bountis are gratefully acknowledged.
*Permanent address: Institute for Problems of Physics and Technology, 119034 Moscow, Russia

†Permanent address: Bogolyubov Institute for Theoretical Physics, 252143 Kyiv, Ukraine

${ }^{1}$ J. Frenkel and T. Kontorova, Phys. Z. Sowjetunion 13, 1 (1938).

${ }^{2}$ F. C. Frank and J. H. van der Merwe, Proc. R. Soc. London Ser. A 198, 205 (1949).

${ }^{3}$ For a review, see, e.g., A. M. Kosevich, Physical Mechanics of Realistic Crystals (Naukova Dumka, Kyiv, 1981).

${ }^{4}$ See, e.g., A. H. Cottrell, Theory of Crystal Dislocation (Blackie, London, 1964); J. P. Hirth and J. Lothe, Theory of Dislocations (Wiley, New York, 1968).

${ }^{5}$ S. Aubry, in Solitons in Condensed Matter Physics, edited by A. R. Bishop and T. Schneider (Springer, Berlin, 1978), p. 264.

${ }^{6}$ A. R. Bishop, J. A. Krumhansl, and S. E. Trullinger, Physica D 1, 1 (1980).

${ }^{7}$ M. Peyrard and M. D. Kruskal, Physica D 14, 88 (1984).

${ }^{8}$ A. G. Naumovets and Yu. S. Vedula, Surf. Sci. Rep. 4, 365 (1984).

${ }^{9}$ For a review, see, e.g., L. V. Yakushevich, Physica D 79, 77 (1994).
${ }^{10}$ W. T. Sanders, J. Appl. Phys. 36, 2822 (1965).

${ }^{11}$ J. Pouget, S. Aubry, A. R. Bishop, and P. S. Lomdahl, Phys. Rev. B 39, 9500 (1989).

${ }^{12}$ J. M. Tamga, M. Remoissenet, and J. Pouget, Phys. Rev. Lett. 75, 357 (1995).

${ }^{13}$ P. S. Lomdahl and D. J. Srolovitz, Phys. Rev. Lett. 57, 2702 (1986).

${ }^{14}$ D. J. Srolovitz and P. S. Lomdahl, Physica D 23, 402 (1986).

${ }^{15}$ O. M. Braun and Yu. S. Kivshar, Phys. Rev. B 44, 7694 (1991).

${ }^{16}$ O. M. Braun, O. A. Chubykalo, Yu. S. Kivshar, and L. Vázquez, Phys. Rev. B 48, 3734 (1993).

${ }^{17}$ A. V. Zolotaryuk, P. L. Christiansen, and A. V. Savin, Phys. Rev. E 54, 3881 (1996).

${ }^{18}$ In our notations, the solitary wave is referred to as a kink (antikink) solution if its profile is an increasing (decreasing) function. In other words, the kink describes a vacancy, i.e., a rarefaction in the chain, while the antikink corresponds to an excess particle in the chain, i.e., its compression.

${ }^{19}$ J. C. Eilbeck and R. Flesch, Phys. Lett. A 149, 200 (1990).

${ }^{20}$ D. B. Duncan, J. C. Eilbeck, H. Feddersen, and J. A. D. Wattis, Physica D 68, 1 (1993). 
${ }^{21}$ P. V. Hobbs, Ice Physics (Clarendon, Oxford, 1974).

${ }^{22}$ St. Pnevmatikos, A. V. Savin, and A. V. Zolotaryuk, in Nonlinear Coherent Structures, edited by M. Barthes and J. Léon (Springer, Berlin, 1989), p. 83.
${ }^{23}$ St. Pnevmatikos, A. V. Savin, and A. V. Zolotaryuk, in Proton Transfer in Hydrogen-Bonded Systems, edited by T. Bountis (Plenum, New York, 1992), p. 79. 